\title{
Active Cancellation System of Acoustic Noise in MR Imaging
}

\author{
Casper K. Chen, Student Member, IEEE, Tzi-Dar Chiueh,* Member, IEEE, and Jyh-Horng Chen
}

\begin{abstract}
In this paper, we introduce a new neural-network architecture for reducing the acoustic noise level in magnetic resonance (MR) imaging processes. The proposed neural network (NN) consists of two cascaded time-delay NN's (TDNN's). This $\mathrm{NN}$ is used as the predictor of a feedback active noise control (ANC) system for reducing acoustic noises. Experimental results with real MR noises show that the proposed system achieved an average noise power attenuation of $18.75 \mathrm{~dB}$, which compares favorably with previous studies. Preliminary results also show that with the proposed ANC system installed, acoustic MR noises are greatly attenuated while verbal communication during MRI sessions is not affected.
\end{abstract}

Index Terms-Active noise cancellation, backpropagation algorithm, magnetic resonance (MR), multilayer perceptrons, timedelay neural networks (TDNN's).

\section{INTRODUCTION}

$\mathbf{T}$ HE invention of magnetic resonance has resulted in a tremendous advance in the medical diagnosis technology. However, as a by-product, the loud acoustic noise generated by the switching gradient currents in MR imagers is a problem to patients undergoing MR imaging (MRI), particularly to children and elder ones. According to U.S. FDA, MR product reclassification documents and product summary data sheets submitted by various MR unit manufacturers, the noise generated by clinical MRI units ranges from $65-95 \mathrm{~dB}$. Such loud sound is not only an annoyance to patients undergoing MRI, but also an interference to the communication between the operators and the patients. Recent research reported the possibility of auditory damage resulting from this acoustic noise of MRI [1], [2]. Temporary threshold shifts of hearing is likely to occur especially for patients who undergo long periods of imaging. Recently, there is a great demand to obtain a true "acoustic noise-free" brain activation mapping without this annoying mechanical noise during brain activation task (i.e., visual stimulus) experiment. With active noise cancellation, an "acoustic noise-free" environment can be created inside MR imagers.

Conventionally, passive sound absorbers are used to suppress acoustic noise levels. However, this method generally does not work well for low-frequency noises whose wavelengths are large compared to the thickness of a typical

Manuscript received August 27, 1997; revised June 24, 1998. Asterisk indicates corresponding author.

C. K. Chen and J.-H. Chen are with the Department of Electrical Engineering, National Taiwan University, Taipei, Taiwan 10617.

*T.-D. Chiueh is with the Department of Electrical Engineering, National Taiwan University, Taipei, Taiwan 10617 (e-mail: chieuh@cc.ee.ntu.edu.tw). Publisher Item Identifier S 0018-9294(99)00828-9.

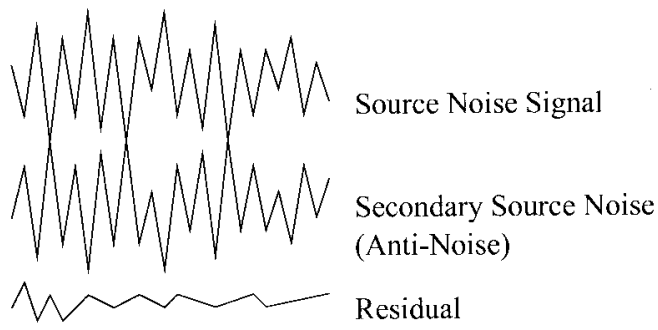

Fig. 1. Noise, antinoise, and residual signals of an ANC system.

acoustic absorber [3]. Active noise control (ANC) was then widely employed to suppress acoustic noises. The idea of active sound control was first introduced by Lueg in 1936 [4]. He measured the sound field with a microphone, electrically manipulated the resultant signal to generate a secondary signal, and then fed it to a loudspeaker to create an interfering sound. According to the superposition principle, the acoustic pressure can then be driven to zero, if the amplitude and phase of the secondary signal are properly adjusted relative to the original signal. Fig. 1 shows the idea of active noise cancellation by illustrating a source noise with a secondary signal and the residual signal.

Traditionally, ANC systems were realized with adaptive digital filters including finite impulse response (FIR) and infinite impulse response (IIR) filters. Although these kinds of ANC systems are usually good at handling low-frequency and narrow-band noises [5]-[10], they have some drawbacks. For example, FIR filters generally do not work well with broad-band noises and IIR filters may become unstable in the adaptation process. Besides, both FIR and IIR filters require custom design for individual noise environment, which is undesirable in practical applications.

A comparably new implementation of ANC is neural networks (NN's). NN's have been applied to speech recognition and speech preprocessing in many previous studies [11]-[15]. A NN speech preprocessor was reported to enhance the signal-to-noise ratio of speech signals and improve speech recognition rate, and the results were encouraging. However, previous works on applying NN to reduce the noise generated by MR imager are not promising. Using the active noise cancellation method in conjunction with a high-quality passive attenuator only produce a noise reduction of less than $10 \mathrm{~dB}$ [16].

In this paper, we present a new cascaded multilayer perceptron NN for active cancellation of acoustic MR imager noise. We concentrate on improving the performance of $\mathrm{NN}$ 


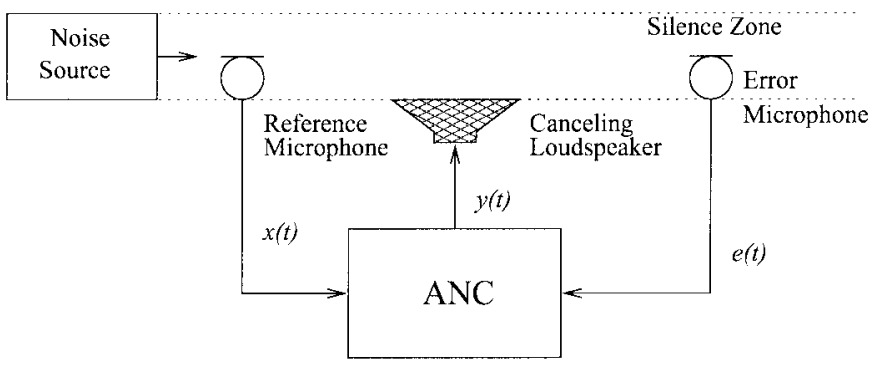

Fig. 2. A feedforward ANC system cancels noise in an air-conditioning duct.

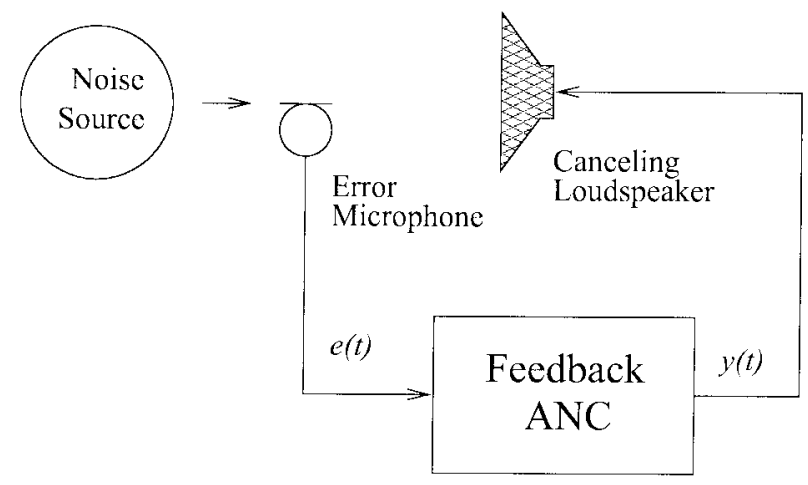

Fig. 3. Drawing of the feedback ANC system.

implementation for active noise cancellation. Without help of any attenuators, we achieved an average noise power reduction of $18.75 \mathrm{~dB}$. Subsequent sections of this paper are organized as follows. Section II describes the ANC system we used in this research. In Section III, the proposed cascaded time-delay neural networks (TDNN's) are presented in detail. Experimental results of the proposed ANC system on acoustic MR noises are illustrated in Section IV, and conclusions are given in Section V.

\section{The Adaptive Noise CANCEllation System}

There are two types of ANC algorithms. The first algorithm is called feedforward control. This algorithm derives a canceling signal from original noise source. Injecting this canceling signal into the noise propagation path, a silence zone can then be created. Fig. 2 shows an example of the feedforward ANC implementation. Note that the use of a reference sensor, which provides rich and well-correlated information of the noise source, is particularly crucial for an effective feedforward ANC system. This requirement prevents its applications from many cases in which the original noise is mixed with the canceling noise (antinoise) and cannot be extracted. On the other hand, another algorithm, called feedback control, does not have this limitation. The feedback control algorithm uses only one sensor to measure the residual noise, which is needed in generating the canceling signal [17]. We chose to implement the ANC system using a feedback configuration, since it is difficult to place a single reference sensor close to acoustic MR noise sources, which are distributed along the magnet.

A diagram of the proposed feedback-adaptive noise cancellation system is shown in Fig. 3. The error microphone acquires the residual noise signal (or error signal) $e(t)$ and

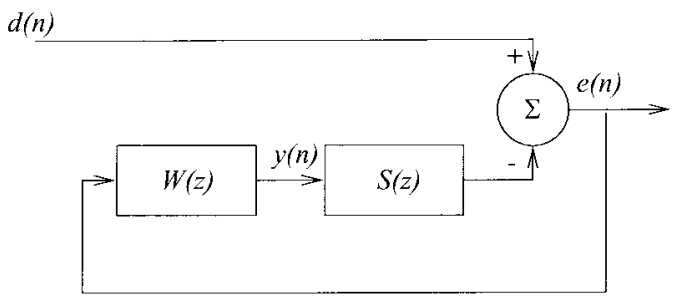

Fig. 4. Block diagram of the feedback ANC system.

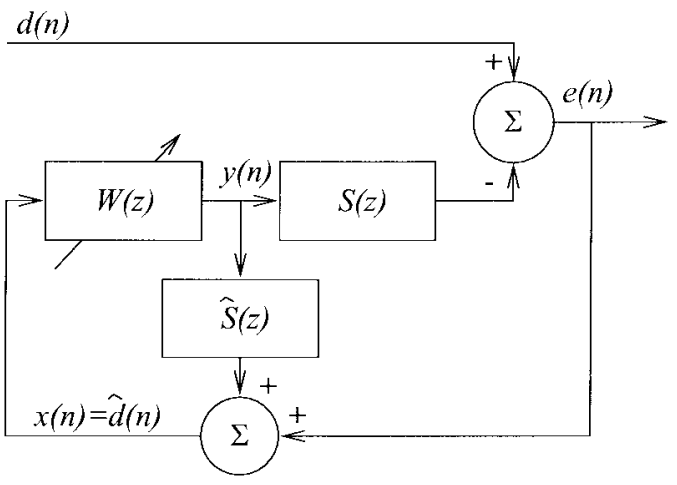

Fig. 5. Adaptive feedback ANC using reference signal synthesized from $y(t)$ and $e(n)$ signals.

provides it to the system as an input, the ANC system then processes $e(t)$ to generate the canceling signal $y(t)$. Fig. 4 shows a discrete-time implementation of the ANC system. In this diagram, $e(n)$ is the $n$th sample of the residual noise measured by the error microphone and $d(n)$ denotes the corresponding sample of the source noise at the location of the microphone. The control signal of the canceling loudspeaker is denoted as $y(n)$, while $W(z)$ represents the adaptive linear filter. $S(z)$ is the transfer function of the transmission path from the loudspeaker to the microphone, which we assume to be linear and time invariant.

Since under perfect cancellation $e(n)$ is supposedly very small, random, and uncorrelated with $d(n)$, it is difficult to generate an antinoise of $d(n)$ from $e(n)$. Hence, the antinoise of $d(n)$ should be generated from $d(n)$ or its estimate, $\hat{d}(n)$. With the residual noise expressed as $E(z)=D(z)-S(z) Y(z)$ in $z$-domain, the source noise is written as $D(z)=E(z)+$ $S(z) Y(z)$. An estimation of the source noise can then be derived as $\hat{D}(z)=E(z)+Y(z) \hat{S}(z)=X(z)$, where $\hat{S}(z)$ is an approximation of $S(z)$. Note that $X(z)$ is now the input signal of the adaptive filter shown in Fig. 5. To ensure convergence of the adaptive filter, we employed the filtered-X least-mean-square (FXLMS) algorithm to update the weights of the adaptive filter $W(z)$ [18]. The block diagram is illustrated in Fig. 6.

The transfer function of the cancellation signal feedback loop $S(z)$ is contributed by three major factors: the frequency response of the error microphone, the processing time delay of the system, and the distortion of the canceling loudspeaker. If we assume that the microphone and the speaker are ideal, then $S(z)$ can be modeled by a pure delay $z^{-\Delta}$. Therefore, we can consider the adaptive filter $W(z)$ as an adaptive predictor. Its function is to generate a predicted signal of the primary noise 


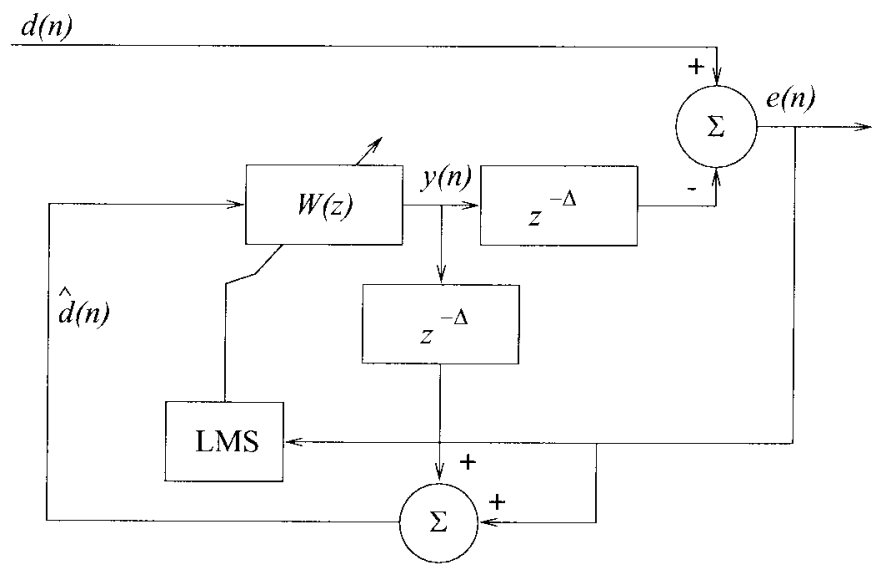

Fig. 6. Adaptive feedback ANC system using the FXLMS algorithm.

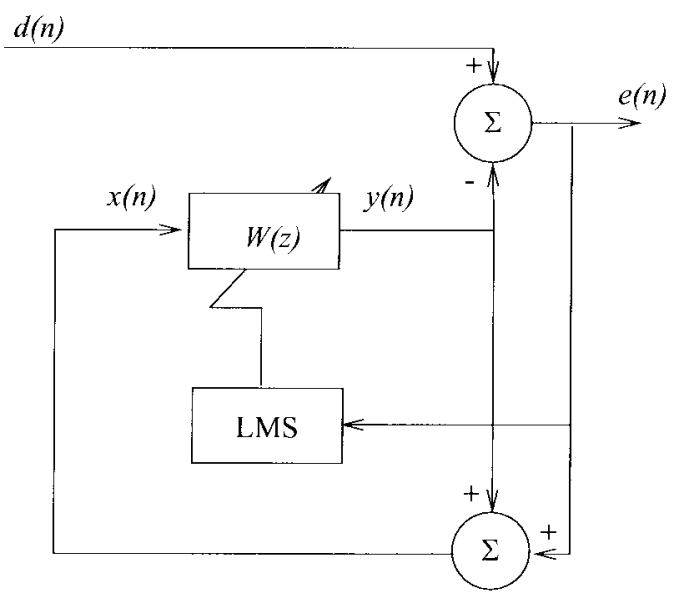

Fig. 7. Block diagram of the proposed ANC system.

$d(n)$ from the reference signal $x(n)$. The predicted signal $y(n)$ is, thus, used to minimize the residual noise $e(n)$. It is obvious that the performance of the adaptive feedback ANC system depends on the accuracy of the prediction. Note that $S(z)$ and $\hat{S}(z)$ are approximated by delays, so they can be absorbed by $W(z)$. The final block diagram of the feedback ANC system is shown in Fig. 7.

\section{THE CASCADED TDNN'S}

ANC systems for MRI rooms require a special feature. Speech signals for communication between patients and MR operators should be clearly preserved while the MR noise is canceled. Traditional adaptive digital filters may be able to procure good noise attenuation, but they also weaken speech signals. In our ANC system, we utilized a NN as the nonlinear adaptive filter. NN's are massively parallel distributed processors that resemble brains in two respects. First, interneuron connection strengths, known as synaptic weights, are used to store knowledge. Second, knowledge is accumulated by the nonlinear network through a learning process. We believe these two characteristics give NN's the ability of suppressing only specific signals as desired by the designer. Generally speaking, there are many possible architectures of NN's, differing in how neurons are organized and the learning algorithm, which is the way synaptic weights

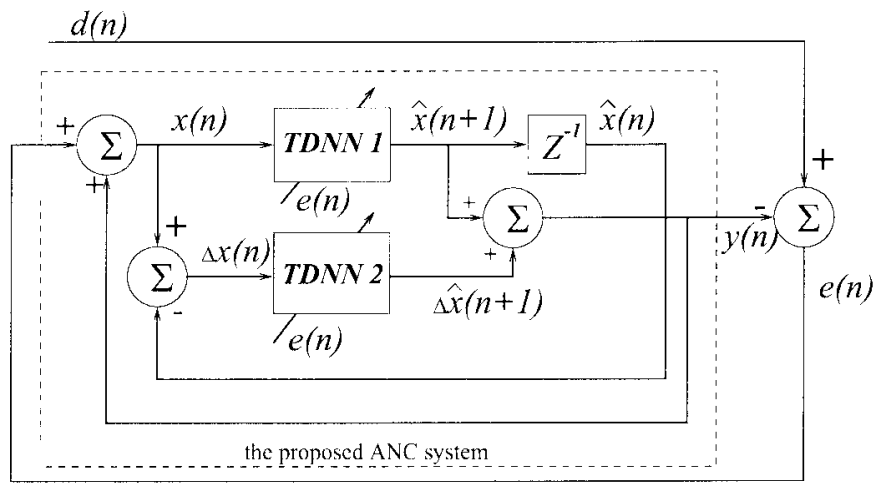

Fig. 8. Two TDNN's are cascaded to implement the proposed ANC system.

are updated. The proposed NN employed a popular structure named time-delay neural networks. The TDNN is a multilayer perceptrons (MLP's) NN whose inputs are formed from a time delay line. Neurons are organized into layers and the input signal propagates through the network layer by layer.

We cascade two TDNN's to accomplish our ANC system as shown in Fig. 8. The cascaded TDNN's perform the function of adaptive filter $W(z)$ in the adaptive feedback ANC system. TDNN 1 performs the fundamental "prediction" of the noise and TDNN 2 forecasts the prediction error of TDNN 1 and compensates for it to improve prediction accuracy. Detail signal descriptions are as follows. TDNN 1 carries out "prediction" of the input signal $x(n)$, which is an estimate of the source noise with compensations for the distortions caused by microphone and loudspeaker. Signal $\hat{x}(n+1)$ is an estimate of signal $x(n+1)$ with a prediction error of $\Delta x(n+1)$, where $\Delta x(n+1)=x(n+1)-\hat{x}(n+1)$. Through the delay element $z^{-1}$, signal $\hat{x}(n)$ is derived and is then used to generate $\Delta x(n)$, which is the previous prediction error of TDNN 1. TDNN 2 then predicts the error of TDNN 1 with this information and outputs $\Delta \hat{x}(n+1)$ for compensation. The output of the ANC system is

$$
y(n)=\hat{x}(n+1)+\Delta \hat{x}(n+1)
$$

This cascaded TDNN architecture has two advantages. First, this architecture could achieve a noise attenuation level about 2 $\mathrm{dB}$ higher than a single TDNN system. A single TDNN system can only obtain 16- to 17-dB noise attenuation even with much larger number of neurons in the TDNN. This architecture also provides flexibility in the network training process. We split the training process of the proposed system into two phases. The first phase is an off-line supervised training for the TDNN 1 with TDNN 2 disabled. The error backpropagation algorithm [19] is employed. The training set is generated from recorded acoustic MR noise. Each input pattern consists of ten samples and the target output is the eleventh sample. This makes TDNN 1 a predictor of $d(n)$ from $d(n-1) \cdots d(n-10)$. Therefore, even without TDNN 2, the ANC system still performs active noise cancellation. This learning process is done iteratively until the synaptic weights and threshold levels of the network stabilize and the average squared error over the training data converges to a minimum value. The second phase of the 
training is to train the TDNN 2 on-line with the synaptic weights of the TDNN 1 fixed.

This training arrangement has several advantages. By training TDNN 1 off line, one can use the same prerecorded data set over and over in each training iteration, which ensures faster convergence for TDNN 1. Also, fixing TDNN 1 weights make the ANC system relatively stable in the TDNN 2 training process. Training TDNN 2 on line provides the system ability to accommodate for minor variations in acoustic MR noises. On-line training means each training data is only used once so that the data need not be recorded, cutting much cost in hardware implementation. With all these advantages, we believe the proposed NN ANC system is more robust than others and more suitable for hardware implementation.

\section{EXPERIMENTAL RESULTS}

We implemented the proposed ANC system using the cascaded TDNN's presented previously. Each of the TDNN's consists of ten input neurons, which takes the input signal and nine previous samples. There are five hidden neurons and one output neuron. Note that the number of input neurons as well as the sampling frequency determine the bandwidth that the ANC system can cover. Experimental results show that the effective noise attenuation can be achieved up to $4000 \mathrm{~Hz}$ with a sampling rate of $22.05 \mathrm{kHz}$.

In the experiments, the source noise was recorded at the National Taiwan University Hospital, Taipei, Taiwan, using gradient-echo-pulse sequence with GE Signa 1.5-T MR imager sampled at $22.05 \mathrm{kHz}$. We split the collected data into two sets: 2000 samples were used for training the NN and the remaining 60000 samples served as the test set. The learning process was fast and the optimum canceler was found within 2000 iterations. This means that the ANC system needs less than $1 \mathrm{~s}$ to adapt itself to a new environment. Fig. 9 illustrates the performance of the ANC system and the waveform of a piece of MR noise used in the experiments. The corresponding antinoise signal generated by the proposed ANC system is shown in Fig. 9(b). The combination of these two signals, the residual signal waveform, is plotted in Fig. 9(c). It is obvious that the proposed system works well in canceling MR noise.

To analyze the bandwidth of the ANC system, we plotted the corresponding spectrum of the MR noises in Fig. 10. The solid line represents the source noise used in the experiments, while the dashed line denotes the residual noise signal. From these two spectra, we notice that a large portion of the MR noise energy lies in low frequencies, where the ANC system achieved very good noise reduction. More experimental results showed that an average noise power attenuation of $18.75 \mathrm{~dB}$ can be achieved. This translates to that $98.65 \%$ of noise power was removed by the ANC network, which is much more effective than other MLP NN's [20].

Furthermore, a piece of source noise signal and its corresponding antinoise signal, which was previously calculated from recorded data, were played with two speakers, respectively. The noise signal was dramatically reduced by the antinoise signal to a level that human ears barely noticed. We then added some human speech to the source noise to generate a new signal. Feeding this new signal to the ANC

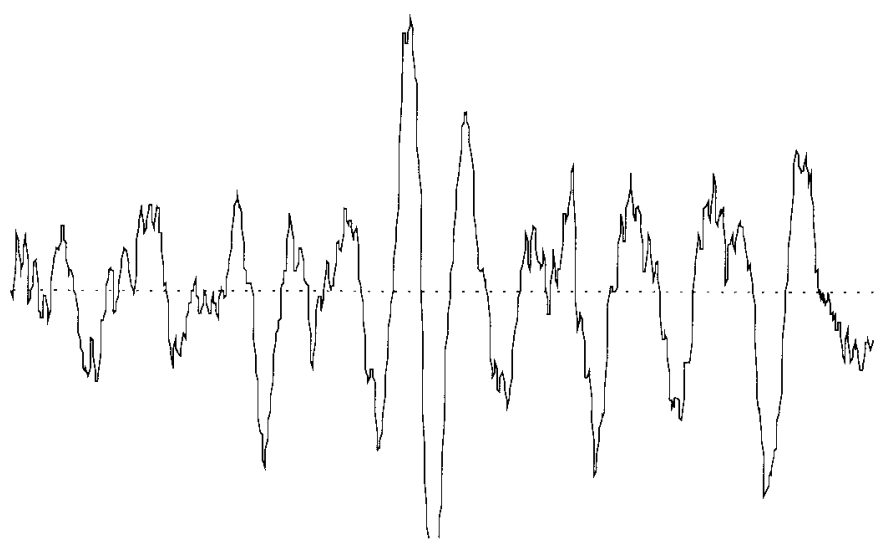

(a)

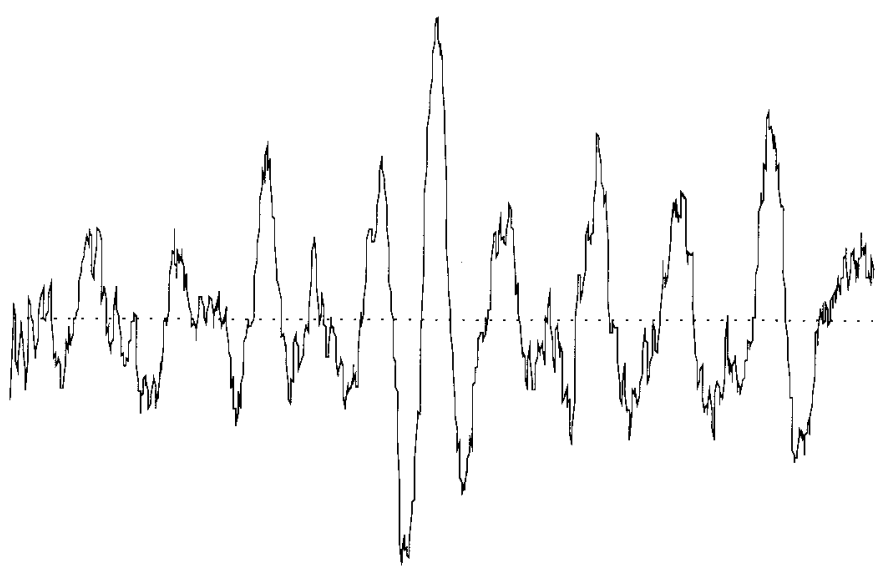

(b)

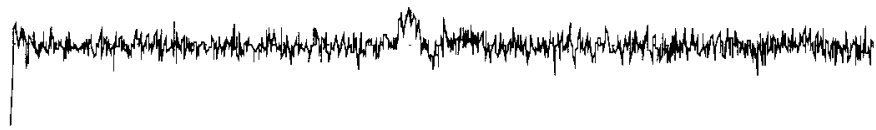

(c)

Fig. 9. (a) Sample waveform of the acoustic noise generated by a MR imager (600 samples), (b) the corresponding antinoise signal waveform, and (c) the residual signal waveform.

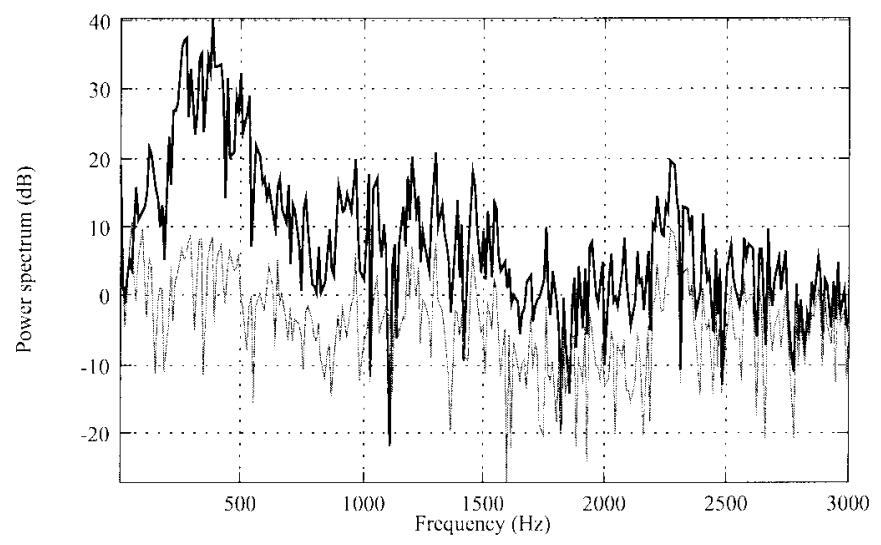

Fig. 10. Spectrum of the noise of the MRI. (Solid line is the source noise and the dashed line is the residual after noise cancellation.)

system, we calculated its antinoise and repeated the above experiment. The result was encouraging because human voice was clearly preserved, while most acoustic MR noises were removed. Fig. 11 shows the ANC system's ability to remove 


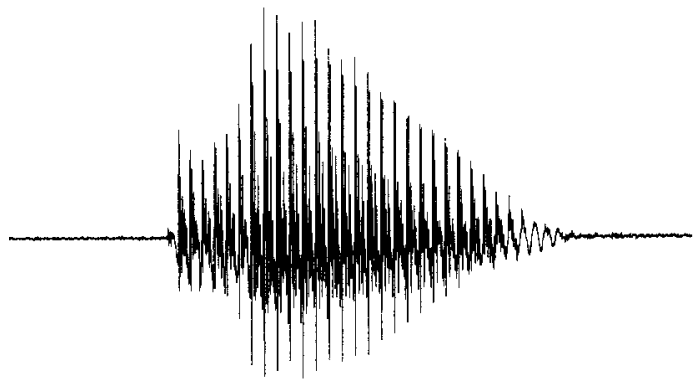

(a)

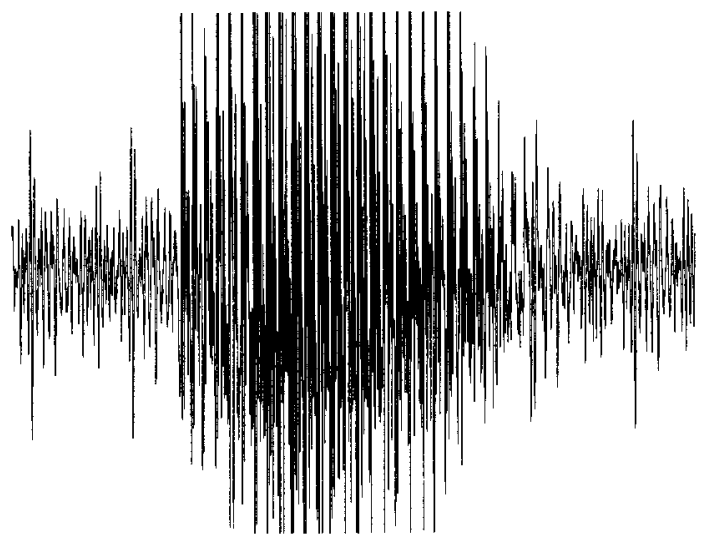

(b)

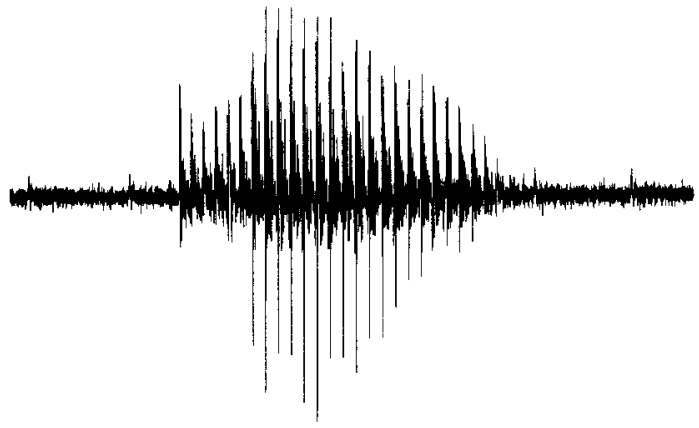

(c)

Fig. 11. (a) Speech signal pronouncing the vowel "a," (b) speech signal corrupted by the MR noise with equal power, and (c) residual signal.

MR noise coupled with human speech signals. The waveform in Fig. 11(a) is a piece of human speech pronouncing vowel "a." Adding an acoustic MR noise to this voice with equal power, we have the corrupted waveform shown in Fig. 11(b). We then fed this corrupted signal to the ANC system. A clean waveform, shown in Fig. 11(c), was then obtained. Note how well the output waveform matches the original speech signal. We further added a segment of English/Chinese broadcasting speech from Voice of America to the MR noise source. Feeding it through the ANC system, we have the noise canceled with the speech nicely preserved and clearly recognized.

\section{CONCLUSION}

In this paper, we proposed a new cascaded TDNN for active cancellation of MR noises. This system has a number of advantages over conventional ANC systems. First, traditional ANC systems must be tailored to each noise environment, but our NN approach is adaptive and able to adapt itself to various noise environments, normally within $1 \mathrm{~s}$. Moreover, even though conventional ANC systems are good at removing pure tone and narrow-band noises, the proposed $\mathrm{NN}$ can deal with broad-band noises in most real-life environments. We believe the proposed TDNN ANC system can create an "acoustic noise-free" environment for functional MRI study and form a solid foundation for future MR noise attenuation systems and other noise reduction systems. Active noise cancellation experiments for the strong burst noise in echo planar imaging (EPI) are under investigation and, presumably, our system will achieve similar remarkable reduction result since EPI noise is also stationary and periodic in time.

\section{REFERENCES}

[1] R. E. Brummett, J. M. Talbot, and P. Charuhas, "Potential hearing loss resulting from MR imaging," Radiol., vol. 169, pp. 539-540, 1988.

[2] E. Kanal, F. G. Shellock, and L. Talagala, "Safety considerations in MR imaging," Radiol., vol. 176, pp. 593-606, 1990.

[3] S. J. Elliott and P. A. Nelson, "Active noise control," IEEE Signal Processing, Mag., Oct. 1993, pp. 12-35.

[4] P. Lueg, "Process of silencing sound oscillations." U.S. Patent 2043416 , 1936.

[5] B. Widrow and S. D. Stearns, Adaptive Signal Processing. Englewood Cliffs, NJ: Prentice-Hall, 1985.

[6] J. N. Denenberg, "Anti-noise," IEEE Potentials, Mag., vol. 11, pp. 36-40, Apr. 1992.

[7] S. J. Elliott, I. M. Stothers, and P. A. Nelson, "A multiple error LMS algorithm and its application to the active control of sound and vibration," IEEE Trans. Acoustics, Speech, Signal Processing, vol. ASSP-35, pp. 1423-1434, Oct. 1987.

[8] A. C. Orgren, S. Dasgupt, C. E. Rohrs, and N. R. Malik, "Noise cancellation with improved residuals," IEEE Trans. Signal Processing, vol. 39, pp. 2629-2639, Dec. 1991.

[9] L. J. Eriksson, M. C. Allie, and R. A. Greiner, "The selection and application of an IIR adaptive filter for use in active sound attenuation," IEEE Trans. Acoustics, Speech, Signal Processing, vol. ASSP-35, pp. 433-437, Apr. 1987.

[10] M. Feder, A. V. Oppenheim, and E. Weinstein, "Maximum likelihood noise cancellation using the EM algorithm," IEEE Trans. Acoustics, Speech, Signal Processing, vol. 37, pp. 204-216, Feb. 1989.

[11] S. Tamura and A. Waibel, "Noise reduction using connectionist models," in Proc. IEEE Int. Conf. Acoustic, Speech, and Signal Processing, New York, 1988, pp. 553-556.

[12] J. L. Elman and D. Zipser, "Learning the hidden structure of speech," J. Acoust. Soc. Am., vol. 83, pp. 1615-1626, Apr. 1988.

[13] F. Xie and D. V. Compernolle, "A family of MLP-based nonlinear spectral estimators for noise reduction," in Proc. IEEE Int. Conf. Acoustic, Speech, and Signal Processing, Adelaide, South Australia, vol. 2, pp. 53-56, 1994.

[14] Y. Gao and J.-P. Haton, "A hierarchical LPNN network for noise reduction and noise degraded speech recognition," in IEEE Int. Conf. Acoustic, Speech, and Signal Processing, Adelaide, South Australia, vol. 2, pp. 89-92, 1994.

[15] J. C. Principe and J.-M. Kuo, "Noise reduction in state space using the focused gamma neural network," in Proc. IEEE Int. Conf. Acoustic, Speech and Signal Processing, Adelaide, South Australia, vol. 2, pp. 533-536, 1994

[16] A. M. Goldman, W. E. Gossman, and P. C. Friedlander, "Reduction of sound levels with antinoise in MR imaging," Radiol., vol. 173, pp. 549-550, 1989.

[17] A. V. Oppenheim, E. Weinstein, K. C. Zangi, M. Feder, and D. Gauger, "Single-sensor active noise cancellation," IEEE Trans. Speech Audio Processing, vol. 2, pp. 285-290, Apr. 1994.

[18] D. R. Morgan, "An analysis of multiple correlation cancellation loops with a filter in the auxiliary path," IEEE Trans. Acoustics, Speech, Signal Processing, vol. ASSP-28, pp. 454-467, Aug. 1980.

[19] S. Haykin, Neural Networks, A Comprehensive Foundation. New York: Macmillan College, 1994.

[20] C K. Chen, T.-D. Chiueh, and J.-H. Chen, "Active cancellation of sound levels with multilayer perceptron neural networks in MR imaging," in Proc. Int. Chinese Forum and 1996 Annu. Meet. of BME Society, Taipei, Taiwan, May 1996, pp. 64-66. 


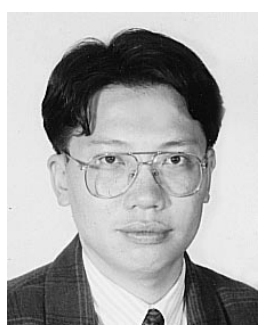

Casper K. Chen (S'92) was born in 1966. He received the B.S. degree from Department of Electrical Engineering, National Central University, Chungli, Taiwan, in 1990. In 1992 he received the M.S. degree from the Institute of Electronics, National Chiao-Tung University Hsinchu, Taiwan. $\mathrm{He}$ received the Ph.D. degree in electrical engineering at National Taiwan University, Taipei, Taiwan, in 1998. His doctoral research includes active noise cancellation (ANC) system design, VLSI design of digital neural network, and artificial neural network for biomedical applications.

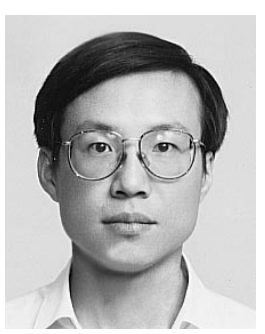

Tzi-Dar Chiueh (S'87-M'90) was born in 1960 $\mathrm{He}$ received the B.S. degree from Department of Electrical Engineering, National Taiwan University (NTUEE), Taipei, Taiwan, in 1983. He then enrolled in California Institute of Technology, Pasadena, in 1985, where he received the Ph.D. degree in 1989.

In 1989 he joined the faculty of NTUEE as an Associate Professor. He became a full Professor in August, 1994. His research interests include VLSI design of analog neuromorphic systems and digital communication systems.

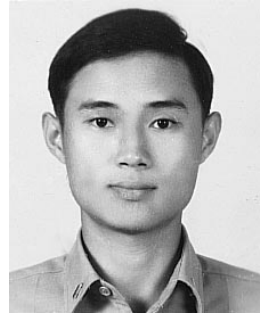

Jyh-Horng Chen was born in 1960. He received the B.S. degree from Department of Electrical Engineering, National Taiwan University (NTUEE), Taipei, Taiwan, in 1983. He then focused his interest on biomedical engineering and received the M.S degree in National Yang Ming University, Taipei, Taiwan. He received the Ph.D degree from the University of California (U.C. Berkeley/U.C. San Francisco) Joint Bioengineering Program.

He joined the faculty of NTUEE as an Associate Professor in 1991. His research interests include magnetic resonance imaging, man-machine interface for disabilities, and medical informatics. Dr. Chen is a member of SMRM and AAPM 